SUPPORTING INFORMATION FOR

\title{
TEMPORAL BIOAVAILABILITY OF ORGANOCHLORINE PESTICIDES AND PCBS
}

\author{
Sethajintanin, D. ${ }^{1}$ and Anderson, K.A ${ }^{1,2}$. \\ ${ }^{1}$ Department of Environmental and Molecular Toxicology \\ ${ }^{2}$ Food Safety and Environmental Stewardship Program, \\ Oregon State University, Corvallis, Oregon 97331
}




\section{Methods}

Sample extraction and analysis. On retrieval, SPMDs were cleaned by gently rubbing with gloved hands in on-site water, then rinsed in $1 \mathrm{~N} \mathrm{HCl}, 18 \mathrm{M} \Omega \cdot \mathrm{cm}$ water, acetone, and isopropanol. Cleaned SPMDs were kept in clean glass amber jars and transported on ice-packs. Samples were stored at $-20{ }^{\circ} \mathrm{C}$ until analysis. PCB congeners and OC pesticides were determined using GC-ECD (Agilent Technologies 6890N Network GC system, Palo Alto, CA) dual capillary columns (db-xlb and db-17 with length $30 \mathrm{~m}$, i.d. $0.25 \mathrm{~mm}$, and film thickness $0.25 \mu \mathrm{m}$, J\&W Scientific Inc., Agilent Technologies, Palo Alto, CA) /dual detectors as previously described (17). Target PCBs include 25 individual congeners included dioxin-like congeners (PCB 77, 105, 114, 118, 126; 156, 169; 189) and non dioxin-like congeners (PCB 37, 44, 49, 52, 60, 74, 87, 99, 101, 128, 138, 153, 166, 170, 180, 183, 187).

Data analysis. The basic theory and mathematical models required for estimation of analyte water concentrations from the concentrations in the SPMD have been described by Huckins et al. (1). The average percent recoveries of PRCs in the deployed SPMDs indicated that the linear uptake model could be assumed for all target analytes in this study. Differences in exposure temperature were corrected by using established SPMD sampling rates $\left(R_{S}\right)$ for PCBs and organochlorine pesticides at multiple temperatures (2-4). The small variations of PRC dissipation rates among sampling sites and under different field seasons suggested the effects of membrane biofouling and flow velocity-turbulence at the membrane surface were negligible.

Estimates of water concentrations $\left(C_{W}\right)$ from SPMD concentrations were calculated by the following equation (1),

$$
C_{W}=C_{S P M D} \cdot M_{S P M D} \cdot R_{s}^{-1} \cdot t^{-1}
$$

where $C_{S P M D}$ is the concentration of the individual analyte in the SPMD, $M_{S P M D}$ is the mass of SPMD in grams, $M_{S P M D}$ is the sampling rate of a standard 1-g triolein SPMD, and $t$ is the time in days.

Precipitation and stream flow data. Precipitation data were obtained from the National Weather Service Forecast Office - Portland, OR (15). Stream flow data recorded at RM 12.8 were obtained from the US Geological Survey (16). Stream flow data were used to convert estimated surface water concentrations into loading estimates 
Calculation of Loads. Stream flow data were used to convert estimated surface water concentrations into loading estimates. The average amount or load of bioavailable DDTs transported in surface water at the lower Willamette River (RM 1-18) during the 4-year study in summer ranged from $1.1 \mathrm{~g} / \mathrm{d}$ to $22 \mathrm{~g} / \mathrm{d}$ for bioavailable $\Sigma$ DDTs; $0.11 \mathrm{~g} / \mathrm{d}$ to $2.1 \mathrm{~g} / \mathrm{d}$ for bioavailable p, p'-DDT; $0.61 \mathrm{~g} / \mathrm{d}$ to 18 $\mathrm{g} / \mathrm{d}$ for bioavailable p,p'-DDD; and $0.36 \mathrm{~g} / \mathrm{d}$ to $2.7 \mathrm{~g} / \mathrm{d}$ for bioavailable p,p'-DDE. The average loads during fall were $2.6 \mathrm{~g} / \mathrm{d}$ to $27 \mathrm{~g} / \mathrm{d}$ for bioavailable $\Sigma$ DDTs; $0.26 \mathrm{~g} / \mathrm{d}$ to $6.9 \mathrm{~g} / \mathrm{d}$ for bioavailable p, p'-DDT; $0.86 \mathrm{~g} / \mathrm{d}$ to $15 \mathrm{~g} / \mathrm{d}$ for bioavailable $\mathrm{p}$, p'-DDD; and $1.3 \mathrm{~g} / \mathrm{d}$ to $6.0 \mathrm{~g} / \mathrm{d}$ for bioavailable $\mathrm{p}, \mathrm{p}$ '-DDE. The highest loads of bioavailable DDTs always observed at RM 7 West during summer and fall. In general, the loads of bioavailable p, p'-DDT and its metabolites were not significantly different between seasons $(p>0.05, t$-test $)$.

\section{Results and Discussion}

River conditions and water chemistry. The river characteristics and water chemistry during passive sampling device deployments are summarized in Supporting Information (SI Table 1). Physicochemical parameters were collected at three river depths. Water chemistry parameters of the three vertical levels were not statistically different within a sampling event. Within the study area (18 miles), there was also no statistical difference between the sampling sites for a given event. The physico-chemical properties were therefore averaged for each event. The difference in water temperature in summer and fall was approximately $10{ }^{\circ} \mathrm{C}$. Dissolved oxygen concentrations ranged from $7.2 \mathrm{mg} / \mathrm{L}$ in summer to $12 \mathrm{mg} / \mathrm{L}$ in fall. The river $\mathrm{pH}$ was neutral. The oxidation-reduction potential tended to decrease in summer $(\mathrm{p}=$ $0.054, t$-test). There was no seasonal pattern observed for specific conductivity, $\mathrm{NH}_{4}{ }^{+}-\mathrm{N}$, and $\mathrm{NO}_{3}{ }^{-}{ }^{-} \mathrm{N}$ concentrations. In addition, the concentrations of TOC, DOC, TSS, and TDS were relatively low and did not change seasonally (Supporting Information Table 2) which suggested that transport of contaminants in the lower Willamette River was primarily in the aqueous phase. Therefore, study of aqueous phase bioavailable contaminant distribution is key to temporal river contaminant characterization 
SI Table 1 Summary of river conditions and water chemistry parameters at Portland Harbor during deployments.

\begin{tabular}{|c|c|c|c|c|c|c|c|c|c|}
\hline $\begin{array}{c}\text { sampling } \\
\text { event }\end{array}$ & $\begin{array}{l}\text { river } \\
\text { flow } \\
\left(\mathrm{ft}^{3} / \mathrm{s}\right)\end{array}$ & $\begin{array}{l}\text { total } \\
\text { rain } \\
\text { (in.) }\end{array}$ & $\begin{array}{l}\text { water } \\
\text { temp. } \\
\left({ }^{\circ} \mathrm{C}\right)\end{array}$ & $\mathrm{pH}$ & $\begin{array}{c}\text { specific } \\
\text { conductivity } \\
(\mathrm{mS} / \mathrm{cm})\end{array}$ & $\begin{array}{c}\mathrm{DO} \\
(\mathrm{mg} / \mathrm{L})\end{array}$ & $\begin{array}{l}\text { ORP } \\
(\mathrm{mV})\end{array}$ & $\begin{array}{l}\mathrm{NH}_{4}{ }^{+}-\mathrm{N} \\
(\mathrm{mg} / \mathrm{L})\end{array}$ & $\begin{array}{l}\mathrm{NO}_{3}{ }^{-}-\mathrm{N} \\
(\mathrm{mg} / \mathrm{L})\end{array}$ \\
\hline 08/02/01- & 7000 & 0 & 22 & 7.4 & 0.12 & 7.2 & 140 & 0.054 & 2.1 \\
\hline 08/16/01 & (170) & & $(0.57)$ & $(0.15)$ & $(0.022)$ & $(0.44)$ & (24) & $(0.097)$ & $(0.24)$ \\
\hline 09/13/01- & 7200 & 0 & 20 & 7.4 & 0.11 & 8.6 & 210 & 0.12 & 2.2 \\
\hline 09/20/01 & (130) & & $(0.19)$ & $(0.17)$ & $(0.022)$ & $(0.43)$ & (23) & $(0.030)$ & $(0.19)$ \\
\hline 10/16/01- & 13000 & 2.93 & 13 & 7.4 & 0.096 & 11 & 200 & 0.14 & 1.4 \\
\hline $11 / 06 / 01$ & $(4000)$ & & $(0.40)$ & $(0.13)$ & $(0.034)$ & $(0.15)$ & (25) & $(0.046)$ & $(0.24)$ \\
\hline 11/06/01- & 27000 & 3.86 & 10 & 7.4 & 0.12 & 12 & 210 & 0.14 & 1.7 \\
\hline $11 / 26 / 01^{\mathrm{b}, \mathrm{c}}$ & (19000) & & $(0.14)$ & $(0.066)$ & $(0.027)$ & $(0.41)$ & (14) & $(0.024)$ & $(0.18)$ \\
\hline 07/17/02- & 8800 & 0.04 & 22 & 7.4 & 0.17 & 8.7 & 160 & 0.25 & 2.1 \\
\hline 08/07/02 & (190) & & $(0.37)$ & $(0.14)$ & $(0.19)$ & $(0.63)$ & (22) & $(0.14)$ & $(0.66)$ \\
\hline 08/07/02- & 8400 & 0 & 22 & 7.3 & 0.19 & 8.3 & 150 & 0.25 & 2.0 \\
\hline $08 / 21 / 02$ & (180) & & $(0.35)$ & $(0.17)$ & $(0.25)$ & $(0.63)$ & (29) & $(0.18)$ & $(0.70)$ \\
\hline 09/11/02- & 9600 & 0.41 & 19 & 7.4 & 0.098 & 9.3 & 160 & 0.21 & 1.6 \\
\hline $09 / 25 / 02$ & (400) & & $(0.76)$ & $(0.079)$ & $(0.015)$ & $(0.44)$ & (28) & $(0.021)$ & $(0.49)$ \\
\hline 11/07/02- & 11000 & 1.88 & 9.5 & 7.2 & 0.078 & 12 & 160 & 0.18 & 1.3 \\
\hline $11 / 26 / 02$ & (2000) & & $(0.46)$ & $(0.11)$ & $(0.016)$ & $(0.17)$ & (12) & $(0.017)$ & $(0.40)$ \\
\hline 10/01/03- & 10000 & 2.32 & 18 & 7.2 & 0.10 & 10 & 280 & 0.24 & 0.62 \\
\hline $10 / 15 / 03^{b, c}$ & (620) & & $(0.22)$ & $(0.18)$ & $(0.017)$ & $(0.86)$ & (23) & $(0.034)$ & $(0.094)$ \\
\hline $11 / 05 / 03-$ & 14000 & 2.71 & 9.0 & NA & NA & NA & NA & NA & NA \\
\hline $11 / 24 / 03^{\mathrm{c}}$ & $(4800)$ & & (1.0) & & & & & & \\
\hline 07/08/04- & 8300 & 0.04 & 23 & 7.2 & 0.097 & 9.5 & 160 & 0.032 & 0.75 \\
\hline $07 / 29 / 04^{b}$ & (390) & & $(0.85)$ & $(0.10)$ & $(0.011)$ & (1.2) & (28) & $(0.017)$ & $(0.18)$ \\
\hline 08/19/04- & 10000 & 2.35 & 22 & 7.2 & 0.10 & 8.5 & 170 & 0.16 & 0.57 \\
\hline $09 / 09 / 04^{\mathrm{b}, \mathrm{c}}$ & (1700) & & $(0.26)$ & $(0.051)$ & $(0.018)$ & (1.2) & (28) & $(0.028)$ & $(0.12)$ \\
\hline 10/19/04- & 21000 & 2.00 & 9.6 & $6.4^{\mathrm{a}}$ & 0.078 & 12 & 310 & 0.17 & 0.16 \\
\hline $11 / 09 / 04^{\mathrm{c}}$ & $(4700)$ & & (1.0) & $(0.13)$ & $(0.018)$ & $(0.49)$ & (127) & $(0.045)$ & $(0.10)$ \\
\hline
\end{tabular}

values are average of 11 sampling sites; numbers in parenthesis are $1 \mathrm{SD}$; NA - data not available

${ }^{\mathrm{a}}$ lower $\mathrm{pH}$ was measured at River Mile 3.5 East $(\mathrm{pH}=4.2)$ and $\mathrm{RM}$ Mile 7 West $(\mathrm{pH}=5.1)$. There was no overt failure of the probe. These two measurements appeared to be an outlier and we did not include in the average;

${ }^{b}$ sewage overflow occurred before or during sample deployment; ${ }^{c}$ rainstorm during sample deployment 
SI Table 2 Summary results of total organic carbon (TOC), dissolved organic carbon (DOC), total suspended solids (TSS), and total dissolved solids (TDS) at Portland Harbor during deployments.

\begin{tabular}{ccccc}
\hline $\begin{array}{c}\text { sampling } \\
\text { date }\end{array}$ & $\begin{array}{c}\text { TOC } \\
(\mathrm{mg} / \mathrm{L})\end{array}$ & $\begin{array}{c}\text { DOC } \\
(\mathrm{mg} / \mathrm{L})\end{array}$ & $\begin{array}{c}\text { TSS } \\
(\mathrm{mg} / \mathrm{L})\end{array}$ & $\begin{array}{c}\text { TDS } \\
(\mathrm{mg} / \mathrm{L})\end{array}$ \\
\hline $11 / 27 / 01$ & 2.4 & $\mathrm{NA}$ & $\mathrm{NA}$ & $\mathrm{NA}$ \\
& $(0.47)$ & 2.1 & & \\
$11 / 26 / 02$ & 2.2 & $(0.83)$ & $\mathrm{NA}$ & $\mathrm{NA}$ \\
& $(0.81)$ & 1.3 & $\mathrm{NA}$ & $\mathrm{NA}$ \\
$10 / 01 / 03$ & 1.4 & $(0.044)$ & & 120 \\
& $(0.0058)$ & 1.6 & 4.2 & $(24)$ \\
$11 / 05 / 03$ & 1.6 & $(0.050)$ & $(1.2)$ & 66 \\
& $(0.058)$ & 1.8 & 6.8 & $(9.3)$ \\
$07 / 08 / 04$ & 1.7 & $(0.27)$ & $(1.3)$ & 94 \\
& $(0.21)$ & 1.9 & 7.8 & $(30)$ \\
$07 / 29 / 04$ & 1.9 & $(0.17)$ & $(2.6)$ & 86 \\
& $(0.21)$ & 1.8 & 7.8 & $(9.0)$ \\
$08 / 19 / 04$ & 1.7 & $(0.093)$ & $(6.5)$ & 74 \\
& $(0.073)$ & 1.7 & 6.8 & $(9.7)$ \\
$09 / 09 / 04$ & 1.8 & $(0.060)$ & $(3.4)$ & \\
& $(0.076)$ & &
\end{tabular}

- Values are averages of 10 sampling sites (River Mile 1 - 18) in 2001, and 4 sampling sites (River Mile 1, River Mile 7 West, River Mile 7 East, and River Mile 18) in 2002, 2003, and 2004. Spatial differences between sampling sites were not statistically significant, therefore the data were averaged.

- Numbers in parenthesis are $1 \mathrm{SD}$

- NA; data not available 
SI Table 3. Seasonal distribution of DDD:DDE ratios from RM 1-18

\begin{tabular}{|l|c|c|c|c|}
\hline \multicolumn{1}{|c|}{ river mile (RM) } & mean ratio in & mean ratio in & p-value & df \\
& summer & fall & -test $)$ & \\
\hline RM 1 & $2.1 \pm 0.39$ & $1.4 \pm 0.47$ & $<0.001$ & 24 \\
\hline RM 3.5 West & $3.7 \pm 0.87$ & $2.4 \pm 1.4$ & 0.146 & 7 \\
\hline RM 3.5 East & $3.8 \pm 0.99$ & $1.8 \pm 1.2$ & 0.012 & 10 \\
\hline RM 7 West & $6.6 \pm 2.1$ & $2.9 \pm 1.3$ & 0.002 & 11 \\
\hline RM 7 East & $1.9 \pm 0.27$ & $1.0 \pm 0.16$ & $<0.001$ & 38 \\
\hline RM 8 & $2.2 \pm 1.0$ & $1.0 \pm 0.40$ & 0.014 & 11 \\
\hline RM 12 & $2.4 \pm 1.7$ & $0.86 \pm 0.39$ & 0.045 & 10 \\
\hline RM 13 & $1.9 \pm 0.75$ & $0.90 \pm 0.34$ & 0.012 & 10 \\
\hline RM 15 & $1.9 \pm 1.4$ & $0.78 \pm 0.35$ & 0.063 & 11 \\
\hline RM 17 & $1.7 \pm 0.95$ & $0.83 \pm 0.33$ & 0.045 & 11 \\
\hline RM 18 & $1.9 \pm 1.4$ & $0.92 \pm 0.28$ & 0.050 & 21 \\
\hline RM 1- 18 & $2.5 \pm 1.6$ & $1.3 \pm 0.84$ & $<0.001$ & 184 \\
\hline
\end{tabular}


SI Table 4: Estimated river concentrations of bioavailable p, p'-DDT and its metabolites, dieldrin, and $\Sigma$ PCBs (sum of 25 congeners) at the Willamette River during summer and fall of 2001 to 2004. River concentrations were estimated from SPMD concentrations. Deployments of SPMDs were typically 7- to 21-day long.

\begin{tabular}{|c|c|c|c|c|c|c|}
\hline sampling event & $\begin{array}{c}\text { p,p'-DDE } \\
(\mathrm{pg} / \mathrm{L})\end{array}$ & $\begin{array}{c}\text { p,p'-DDD } \\
(\mathrm{pg} / \mathrm{L})\end{array}$ & $\begin{array}{c}\text { p,p'-DDT } \\
(\mathrm{pg} / \mathrm{L})\end{array}$ & $\begin{array}{c}\Sigma \text { DDTs } \\
(\mathrm{pg} / \mathrm{L})\end{array}$ & $\begin{array}{c}\text { dieldrin } \\
\text { (pg/L) }\end{array}$ & $\begin{array}{c}\Sigma \text { PCBs } \\
(\mathrm{pg} / \mathrm{L})\end{array}$ \\
\hline $\begin{array}{l}\text { detection limit } \\
(\mathrm{pg} / \mathrm{L})^{\mathrm{a}}\end{array}$ & $2-15$ & $3-7$ & $3-7$ & & $3-19$ & $0.69-54^{b}$ \\
\hline \multicolumn{7}{|l|}{ August 2001} \\
\hline $\mathrm{RM} 1^{\mathrm{c}}$ & $66(5.3)$ & 167 (49) & $2.3(3.3)$ & $236(51)$ & $43(2.0)$ & $13(11)$ \\
\hline RM 3.5 West & 44 & 126 & 4 & 174 & 47 & 14 \\
\hline RM 3.5 East & 55 & 250 & 5 & 309 & 58 & 31 \\
\hline RM 7 West & 125 & 514 & 48 & 687 & 57 & 31 \\
\hline RM 7 East & NA & NA & NA & NA & NA & NA \\
\hline RM 8 & 48 & 112 & bdl & 161 & 75 & 13 \\
\hline RM 12 & 15 & 59 & bdl & 74 & 42 & 14 \\
\hline RM 13 & 18 & 56 & 5 & 79 & 41 & 18 \\
\hline RM 15 & 11 & 20 & bdl & 31 & 23 & 4.8 \\
\hline RM 17 & 13 & 46 & 13 & 72 & 37 & 8.5 \\
\hline $\mathrm{RM} 18^{\mathrm{c}}$ & $11(4.1)$ & $42(25)$ & $7.5(11)$ & $60(40)$ & $35(15)$ & $10(8.2)$ \\
\hline \multicolumn{7}{|l|}{ September 2001} \\
\hline $\mathrm{RM} 1^{\mathrm{c}}$ & $120(1.5)$ & $289(32)$ & $6.0(8.5)$ & $414(39)$ & $65(2.5)$ & $120(13)$ \\
\hline RM 3.5 West & 66 & 327 & bdl & 393 & 50 & 89 \\
\hline RM 3.5 East & 68 & 371 & bdl & 439 & 55 & 94 \\
\hline RM 7 West & 201 & 956 & 100 & 1257 & 108 & 63 \\
\hline RM 7 East & NA & NA & NA & NA & NA & NA \\
\hline RM 8 & 114 & 481 & bdl & 595 & 71 & 123 \\
\hline RM 12 & 22 & 112 & bdl & 134 & 34 & 12 \\
\hline RM 13 & 28 & 73 & 14 & 115 & 50 & 13 \\
\hline RM 15 & 30 & 144 & 11 & 184 & 38 & 4.2 \\
\hline RM 17 & 33 & 61 & 18 & 111 & 63 & 17 \\
\hline RM $18^{c}$ & $21(0.77)$ & $57(68)$ & $4.6(6.5)$ & $82(60)$ & $45(3.0)$ & $13(4.8)$ \\
\hline \multicolumn{7}{|l|}{ October 2001} \\
\hline $\mathrm{RM} 1^{\mathrm{c}}$ & $82(10)$ & $125(18)$ & $32(3.7)$ & $239(25)$ & $56(2.7)$ & $130(0.35)$ \\
\hline RM 3.5 West & NA & NA & NA & NA & NA & NA \\
\hline RM 3.5 East & NA & NA & NA & NA & NA & NA \\
\hline RM 7 West & 87 & 266 & 168 & 521 & 59 & 72 \\
\hline RM 7 East & NA & NA & NA & NA & NA & NA \\
\hline RM 8 & 33 & 35 & 14 & 83 & 34 & 36 \\
\hline RM 12 & 21 & 25 & 18 & 64 & 40 & 29 \\
\hline RM 13 & 27 & 34 & 18 & 79 & 40 & 26 \\
\hline RM 15 & 23 & 13 & 10 & 45 & 31 & 16 \\
\hline RM 17 & 18 & 20 & 13 & 51 & 26 & 21 \\
\hline RM $18^{\mathrm{c}}$ & $26(0.32)$ & $28(2.8)$ & $16(0.12)$ & $69(2.4)$ & $62(0.44)$ & $35(1.1)$ \\
\hline \multicolumn{7}{|l|}{ November 2001} \\
\hline RM 1 & 71 & 73 & 26 & 170 & 75 & 104 \\
\hline RM 3.5 West & NA & NA & NA & NA & NA & NA \\
\hline RM 3.5 East & 114 & 140 & 76 & 330 & 137 & 270 \\
\hline RM 7 West & 93 & 180 & 76 & 349 & 81 & 94 \\
\hline RM 7 East & NA & NA & NA & NA & NA & NA \\
\hline
\end{tabular}




\begin{tabular}{|c|c|c|c|c|c|c|}
\hline sampling event & $\begin{array}{c}\text { p,p'-DDE } \\
(\mathrm{pg} / \mathrm{L})\end{array}$ & $\begin{array}{c}\text { p,p'-DDD } \\
\text { (pg/L) }\end{array}$ & $\begin{array}{c}\mathrm{p}, \mathrm{p} \text { '-DDT } \\
(\mathrm{pg} / \mathrm{L})\end{array}$ & $\begin{array}{c}\sum \text { DDTs } \\
(\mathrm{pg} / \mathrm{L})\end{array}$ & $\begin{array}{c}\text { dieldrin } \\
(\mathrm{pg} / \mathrm{L})\end{array}$ & $\begin{array}{c}\Sigma \text { PCBs } \\
(\mathrm{pg} / \mathrm{L})\end{array}$ \\
\hline $\begin{array}{l}\text { detection limit } \\
(\mathrm{pg} / \mathrm{L})^{\mathrm{a}}\end{array}$ & $2-15$ & $3-7$ & $3-7$ & & $3-19$ & $0.69-54^{b}$ \\
\hline RM 8 & 50 & 33 & 22 & 104 & 78 & 101 \\
\hline RM 12 & 46 & 27 & 32 & 105 & 83 & 49 \\
\hline RM 13 & 67 & 42 & 26 & 135 & 101 & 100 \\
\hline RM 15 & 56 & 29 & 21 & 106 & 102 & 37 \\
\hline RM 17 & 50 & 30 & 18 & 98 & 104 & 105 \\
\hline RM 18 & NA & NA & NA & NA & NA & NA \\
\hline \multicolumn{7}{|l|}{ July 2002} \\
\hline $\mathrm{RM} 1^{\mathrm{c}}$ & $44(11)$ & $95(6.3)$ & $7.1(0.72)$ & $146(18)$ & $24(1.2)$ & $11(1.7)$ \\
\hline RM 3.5 West & 54 & 159 & 13 & 226 & 54 & 33 \\
\hline RM 3.5 East & 52 & 147 & 14 & 212 & 53 & 52 \\
\hline RM 7 West & 160 & 939 & 143 & 1242 & 71 & 47 \\
\hline RM 7 East & NA & NA & NA & NA & NA & NA \\
\hline RM 8 & 43 & 70 & 13 & 126 & 65 & 35 \\
\hline RM 12 & 25 & 42 & 9 & 76 & 51 & 13 \\
\hline RM 13 & 45 & 68 & 8 & 121 & 74 & 32 \\
\hline RM 15 & 24 & 30 & 8 & 63 & 38 & 6.4 \\
\hline RM 17 & 29 & 31 & 7 & 68 & 50 & 8.5 \\
\hline RM $18^{\mathrm{c}}$ & $18(4.1)$ & $24(6.5)$ & $4.3(1.3)$ & $47(12)$ & $44(15)$ & $8.1(2.7)$ \\
\hline \multicolumn{7}{|l|}{ August 2002} \\
\hline RM 1 & $52(2.5)^{\mathrm{c}}$ & $85(2.6)^{\mathrm{c}}$ & $17(1.8)^{\mathrm{c}}$ & $154(3.4)^{\mathrm{c}}$ & $28(0.97)^{\mathrm{c}}$ & 15 \\
\hline RM 3.5 West & 61 & 218 & 20 & 300 & 62 & 45 \\
\hline RM 3.5 East & 66 & 229 & 24 & 319 & 66 & 60 \\
\hline RM 7 West & 129 & 1207 & 130 & 1465 & 67 & 52 \\
\hline RM 7 East & NA & NA & NA & NA & NA & NA \\
\hline RM 8 & 37 & 63 & 12 & 112 & 48 & 37 \\
\hline RM 12 & 23 & 33 & 10 & 66 & 41 & 19 \\
\hline RM 13 & 25 & 41 & 11 & 76 & 47 & 26 \\
\hline RM 15 & 23 & 33 & 9 & 65 & 46 & 9.3 \\
\hline RM 17 & 23 & 31 & 13 & 67 & 60 & 11 \\
\hline RM $18^{c}$ & $18(4.1)$ & $25(5.3)$ & $10(2.4)$ & $53(12)$ & $41(7.3)$ & $12(2.6)$ \\
\hline \multicolumn{7}{|l|}{ September 2002} \\
\hline $\mathrm{RM} 1^{\mathrm{c}}$ & $49(2.5)$ & $96(18)$ & $11(1.8)$ & $156(22)$ & $32(5.6)$ & $26(2.2)$ \\
\hline RM 3.5 West & NA & NA & NA & NA & NA & NA \\
\hline RM 3.5 East & 52 & 175 & 18 & 245 & 43 & 56 \\
\hline RM 7 West & 90 & 759 & 74 & 923 & 39 & 52 \\
\hline RM 7 East $^{d}$ & $37(10)$ & $68(21)$ & $20(12)$ & $126(39)$ & $27(10)$ & $55(42)$ \\
\hline RM 8 & 27 & 39 & 7 & 74 & 28 & 24 \\
\hline RM 12 & 22 & 25 & 11 & 58 & 28 & 12 \\
\hline RM 13 & 29 & 38 & 11 & 78 & 30 & 22 \\
\hline RM 15 & 21 & 22 & 7 & 49 & 23 & 3.0 \\
\hline RM 17 & 15 & 16 & 8 & 39 & 22 & bdl \\
\hline RM $18^{c}$ & $18(0.61)$ & $22(1.3)$ & $9.8(1.1)$ & $50(1.7)$ & $33(0.14)$ & $9.5(1.6)$ \\
\hline \multicolumn{7}{|l|}{ November 2002} \\
\hline $\mathrm{RM} 1^{\mathrm{c}}$ & $91(3.6)$ & $102(11)$ & $11(1.2)$ & $204(16)$ & $83(8.0)$ & $29(3.5)$ \\
\hline RM 3.5 West & 78 & 108 & 14 & 200 & 69 & 32 \\
\hline RM 3.5 East & 61 & 79 & 14 & 153 & 65 & 37 \\
\hline RM 7 West & 129 & 321 & 55 & 505 & 64 & 30 \\
\hline RM 7 East $^{c}$ & $43(4.8)$ & $41(5.4)$ & $7.6(0.079)$ & $92(10)$ & $50(0.29)$ & $16(3.7)$ \\
\hline
\end{tabular}




\begin{tabular}{|c|c|c|c|c|c|c|}
\hline sampling event & $\begin{array}{c}\text { p,p'-DDE } \\
\text { (pg/L) }\end{array}$ & $\begin{array}{c}\text { p,p'-DDD } \\
\text { (pg/L) }\end{array}$ & $\begin{array}{c}\text { p,p'-DDT } \\
\text { (pg/L) }\end{array}$ & $\begin{array}{c}\sum \text { DDTs } \\
(\mathrm{pg} / \mathrm{L})\end{array}$ & $\begin{array}{c}\text { dieldrin } \\
(\mathrm{pg} / \mathrm{L})\end{array}$ & $\begin{array}{c}\Sigma \text { PCBs } \\
(\mathrm{pg} / \mathrm{L})\end{array}$ \\
\hline $\begin{array}{l}\text { detection limit } \\
(\mathrm{pg} / \mathrm{L})^{\mathrm{a}}\end{array}$ & $2-15$ & $3-7$ & $3-7$ & & $3-19$ & $0.69-54^{b}$ \\
\hline RM 8 & 37 & 28 & 7 & 73 & 46 & 10 \\
\hline RM 12 & 38 & 24 & 8 & 70 & 46 & 5.6 \\
\hline RM 13 & 45 & 35 & 9 & 88 & 53 & 29 \\
\hline RM 15 & 47 & 27 & 9 & 83 & 53 & 4.9 \\
\hline RM 17 & 13 & 7 & 6 & 27 & 13 & bdl \\
\hline RM $18^{\mathrm{c}}$ & $52(8.0)$ & $37(9.2)$ & $10(1.1)$ & $99(18)$ & $84(24)$ & $51(7.3)$ \\
\hline \multicolumn{7}{|l|}{ October 2003} \\
\hline $\mathrm{RM} 1^{\mathrm{c}}$ & $76(12)$ & $170(4.5)$ & $14(1.5)$ & $260(18)$ & $47(2.6)$ & $48(2.6)$ \\
\hline RM 3.5 West & 54 & 242 & 36 & 332 & 54 & 69 \\
\hline RM 3.5 East & 59 & 242 & 37 & 338 & 61 & 102 \\
\hline RM 7 West & 105 & 426 & 149 & 680 & 48 & 61 \\
\hline RM 7 East $^{\mathrm{e}}$ & 47 (8.9) & $51(12)$ & 8.9 (6.9) & $107(25)$ & $40(22)$ & $41(14)$ \\
\hline RM 8 & 24 & 34 & 6 & 65 & 34 & 35 \\
\hline RM 12 & NA & NA & NA & NA & NA & NA \\
\hline RM 13 & NA & NA & NA & NA & NA & NA \\
\hline RM 15.5 & 23 & 26 & bdl & 49 & 35 & 1.5 \\
\hline RM 17 & 13 & 17 & bdl & 30 & 24 & bdl \\
\hline RM 18.5 & 14 & 15 & bdl & 29 & 29 & bdl \\
\hline \multicolumn{7}{|l|}{ November 2003} \\
\hline $\mathrm{RM} 1^{\mathrm{c}}$ & 264 (17) & $223(8.1)$ & $39(9.6)$ & $527(35)$ & $76(5.9)$ & $39(3.5)$ \\
\hline RM 3.5 West & NA & NA & NA & NA & NA & NA \\
\hline RM 3.5 East & 166 & 119 & 37 & 322 & 86 & 80 \\
\hline RM 7 West & 199 & 232 & 102 & 533 & 76 & 22 \\
\hline RM 7 East $^{\mathrm{c}}$ & $67(6.7)$ & $50(6.0)$ & $14(0.53)$ & $131(13)$ & $67(2.8)$ & $15(6.7)$ \\
\hline RM 8 & 70 & 44 & 13 & 127 & 64 & 13 \\
\hline RM 12 & 58 & 26 & 16 & 101 & 60 & bdl \\
\hline RM 13 & 81 & 38 & 20 & 139 & 68 & 5.4 \\
\hline RM 15 & 69 & 32 & 17 & 118 & 74 & 4.3 \\
\hline RM 17 & 53 & 24 & 16 & 93 & 58 & bdl \\
\hline RM 18 & 54 & 21 & 16 & 91 & 62 & 5.5 \\
\hline \multicolumn{7}{|l|}{ July 2004} \\
\hline $\mathrm{RM} 1^{\mathrm{c}}$ & $50(1.9)$ & $102(7.4)$ & $9.0(0.18)$ & $161(9.5)$ & $35(2.7)$ & $90(6.3)$ \\
\hline RM 3.5 West & 57 & 234 & 21 & 311 & 55 & 128 \\
\hline RM 3.5 East & 42 & 133 & 14 & 188 & 47 & 154 \\
\hline RM 7 West & 111 & 804 & 132 & 1046 & 52 & 126 \\
\hline RM 7 East & 29 & 66 & 8 & 103 & 39 & 99 \\
\hline RM 8 & 26 & 54 & 8 & 88 & 45 & 90 \\
\hline RM 12 & 28 & 39 & bdl & 67 & 50 & 89 \\
\hline RM 13 & 28 & 39 & 7 & 75 & 45 & 90 \\
\hline RM 15 & 23 & 28 & 6 & 58 & 43 & 42 \\
\hline RM 17 & 34 & 43 & 9 & 86 & 64 & 34 \\
\hline RM $18^{c}$ & $19(4.4)$ & $22(2.9)$ & $6.0(1.3)$ & $47(8.5)$ & $38(7.6)$ & $42(3.3)$ \\
\hline \multicolumn{7}{|l|}{ August 2004} \\
\hline $\mathrm{RM} 1^{\mathrm{c}}$ & $91(0.52)$ & $126(2.1)$ & $56(1.1)$ & $273(2.7)$ & $68(2.8)$ & $95(2.9)$ \\
\hline RM 3.5 West & 63 & 149 & 25 & 236 & 62 & 152 \\
\hline RM 3.5 East & 52 & 120 & 29 & 201 & 65 & 123 \\
\hline RM 7 West & 106 & 546 & 161 & 813 & 69 & 116 \\
\hline RM 7 East & NA & NA & NA & NA & NA & NA \\
\hline
\end{tabular}




\begin{tabular}{|c|c|c|c|c|c|c|}
\hline sampling event & $\begin{array}{c}\text { p,p'-DDE } \\
(\mathrm{pg} / \mathrm{L})\end{array}$ & $\begin{array}{c}\text { p,p'-DDD } \\
(\mathrm{pg} / \mathrm{L})\end{array}$ & $\begin{array}{c}\text { p,p'-DDT } \\
(\mathrm{pg} / \mathrm{L})\end{array}$ & $\begin{array}{c}\sum \text { DDTs } \\
(\mathrm{pg} / \mathrm{L})\end{array}$ & $\begin{array}{c}\text { dieldrin } \\
(\mathrm{pg} / \mathrm{L})\end{array}$ & $\begin{array}{c}\Sigma \text { PCBs } \\
(\mathrm{pg} / \mathrm{L})\end{array}$ \\
\hline $\begin{array}{c}\text { detection limit } \\
(\mathrm{pg} / \mathrm{L})\end{array}$ & $2-15$ & $3-7$ & $3-7$ & & $3-19$ & $0.69-54^{\mathrm{b}}$ \\
\hline RM 8 & 30 & 50 & 11 & 91 & 67 & 104 \\
\hline RM 12 & 25 & 36 & 13 & 74 & 68 & 69 \\
\hline RM 13 & 28 & 37 & 18 & 83 & 60 & 92 \\
\hline RM 15 & 31 & 43 & 17 & 90 & 68 & 51 \\
\hline RM 17 & 19 & 20 & 8 & 48 & 51 & 35 \\
\hline RM 18 & $23(0.23)$ & $29(1.0)$ & $15(5.2)$ & $67(4.0)$ & $60(2.0)$ & $53(4.6)$ \\
\hline October 2004 & & & & & & $260(3.1)$ \\
\hline RM 1 & $122(1.1)$ & $139(3.0)$ & $28(0.27)$ & $290(4.4)$ & $108(1.5)$ & 263 \\
\hline RM 3.5 West & 70 & 104 & 29 & 203 & 71 & 152 \\
\hline RM 3.5 East & 82 & 98 & 29 & 209 & 93 & 408 \\
\hline RM 7 West & 282 & 656 & 403 & 1341 & 105 & 184 \\
\hline RM 7 East & 31 & 30 & 8 & 69 & 66 & 63 \\
\hline RM 8 & 45 & 41 & 10 & 96 & 85 & 95 \\
\hline RM 12 & 32 & 28 & 10 & 71 & 69 & 64 \\
\hline RM 13 & 56 & 53 & 21 & 129 & 84 & 162 \\
\hline RM 15 & 49 & 39 & 10 & 98 & 86 & 46 \\
\hline RM 17 & 37 & 27 & 9 & 73 & 86 & 49 \\
\hline RM 18 & $58(0.58)$ & $47(0.93)$ & $18(1.2)$ & $124(2.7)$ & $123(0.90)$ & $68(3.2)$ \\
\hline
\end{tabular}

$\mathrm{n}=1$ composite sample of 5 SPMDs; duplicate samples were at River Mile 1 and 18; values in parenthesis are 1SD; bdl- below detection limit; NA-data not available

${ }^{a}$ range of method detection limits from 13 sampling events

${ }^{\mathrm{b}}$ detection limits for 25 individual PCB congeners

${ }^{\mathrm{c}} \mathrm{n}=2$

${ }^{\mathrm{d}} \mathrm{n}=19$

${ }^{\mathrm{d}} \mathrm{n}=15$ 
SI Figure 1. Estimated concentrations of bioavailable p, p'-DDT and its metabolites in surface water at River Mile 7 West at Portland Harbor, Oregon in relation to average water temperature, average river flow, total precipitation, total organic carbon (TOC, black circle), and dissolved organic carbon (DOC, white circle) concentrations

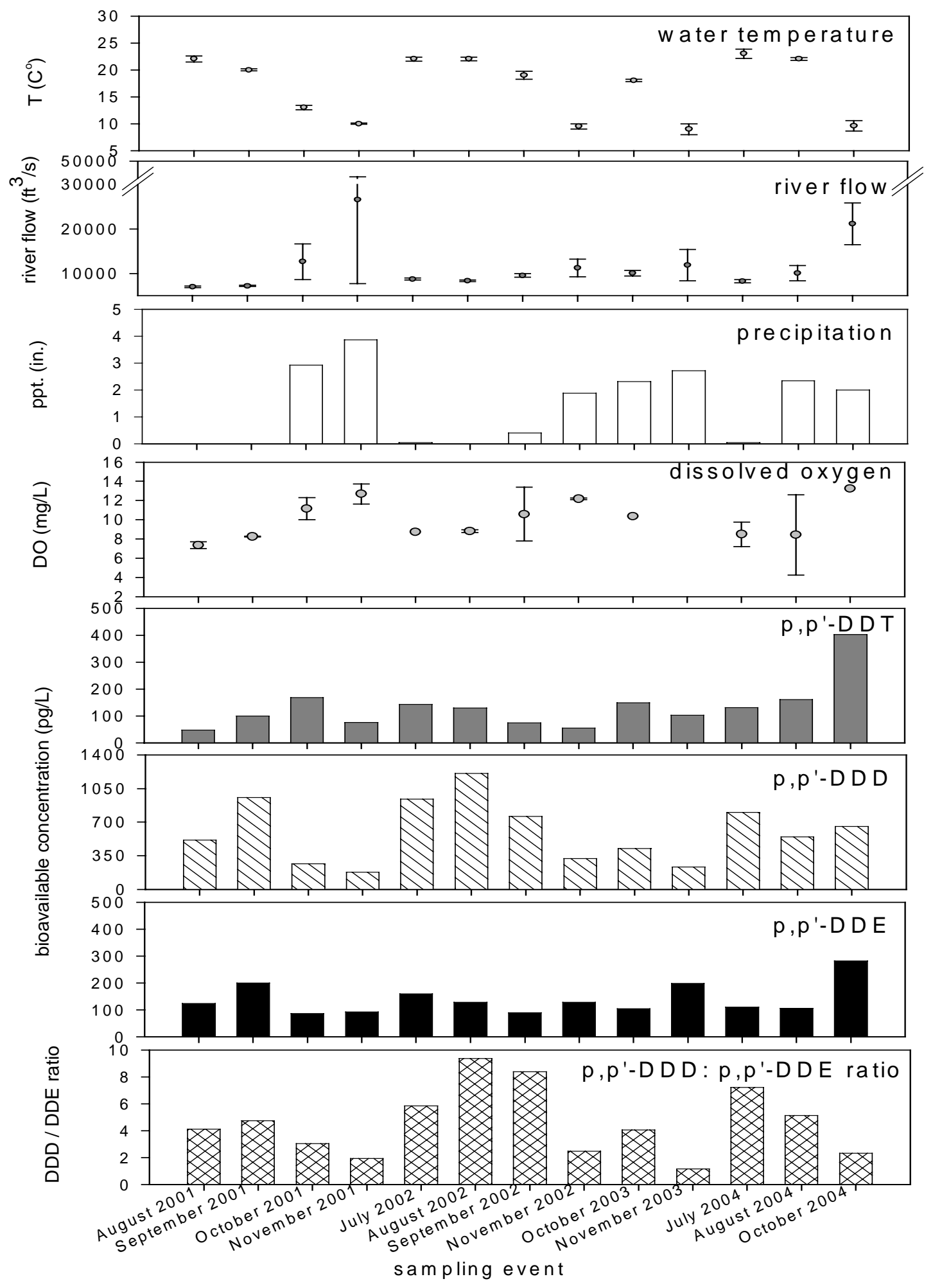


SI Figure 3. Average percent contributions of PCB congeners in surface water at the lower Willamette River during summer and fall in 2001 to 2004. The error bars denoted 1 SD
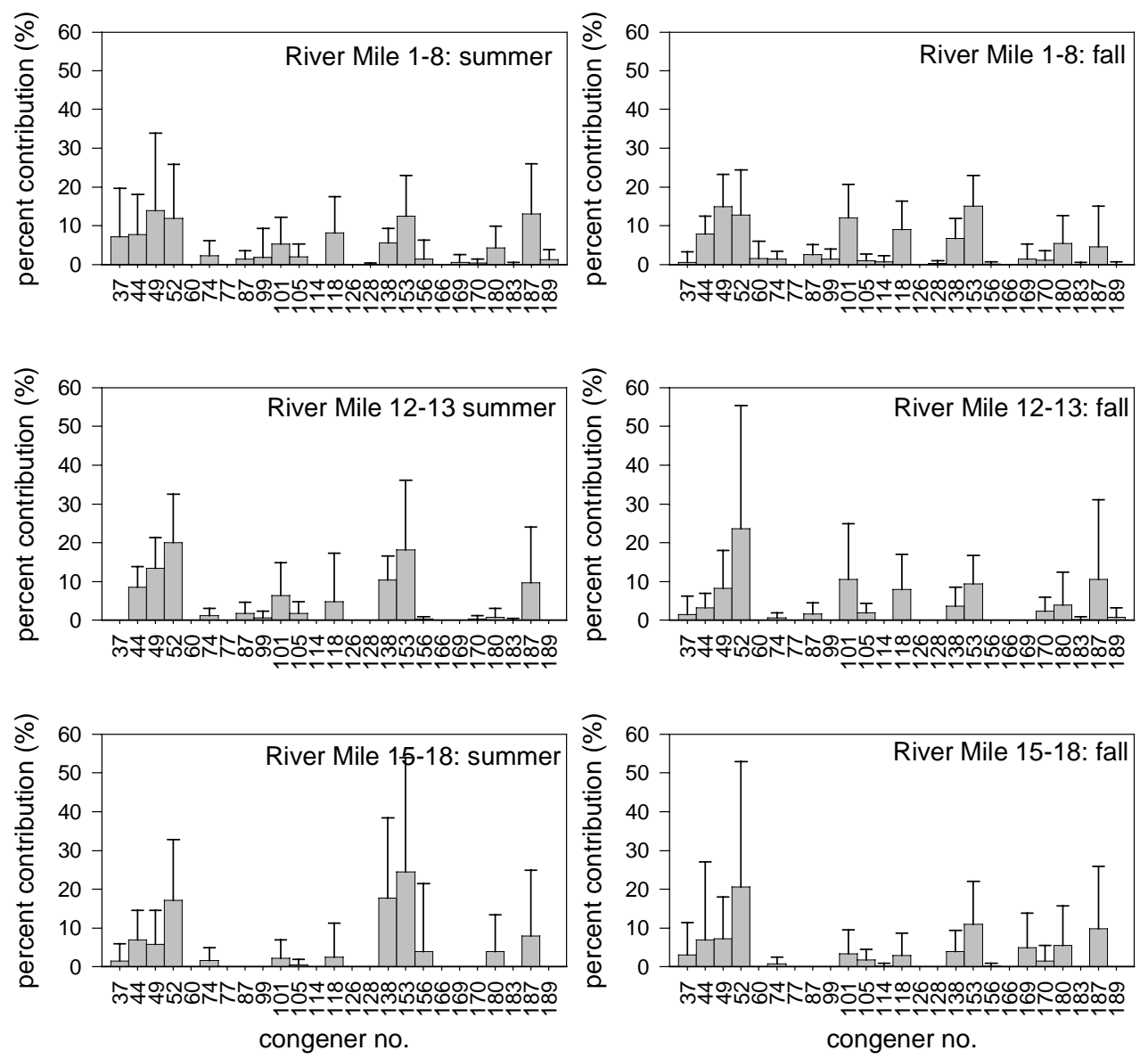
SI Figure 4 Average ratios of PCB homolog to bioavailable $\Sigma$ PCBs (sum of 25 individual PCB congeners) in surface water at the lower Willamette River during summer and fall in 2001 to 2004. The error bars denoted 1 SD. See detail of PCB homologs in text.
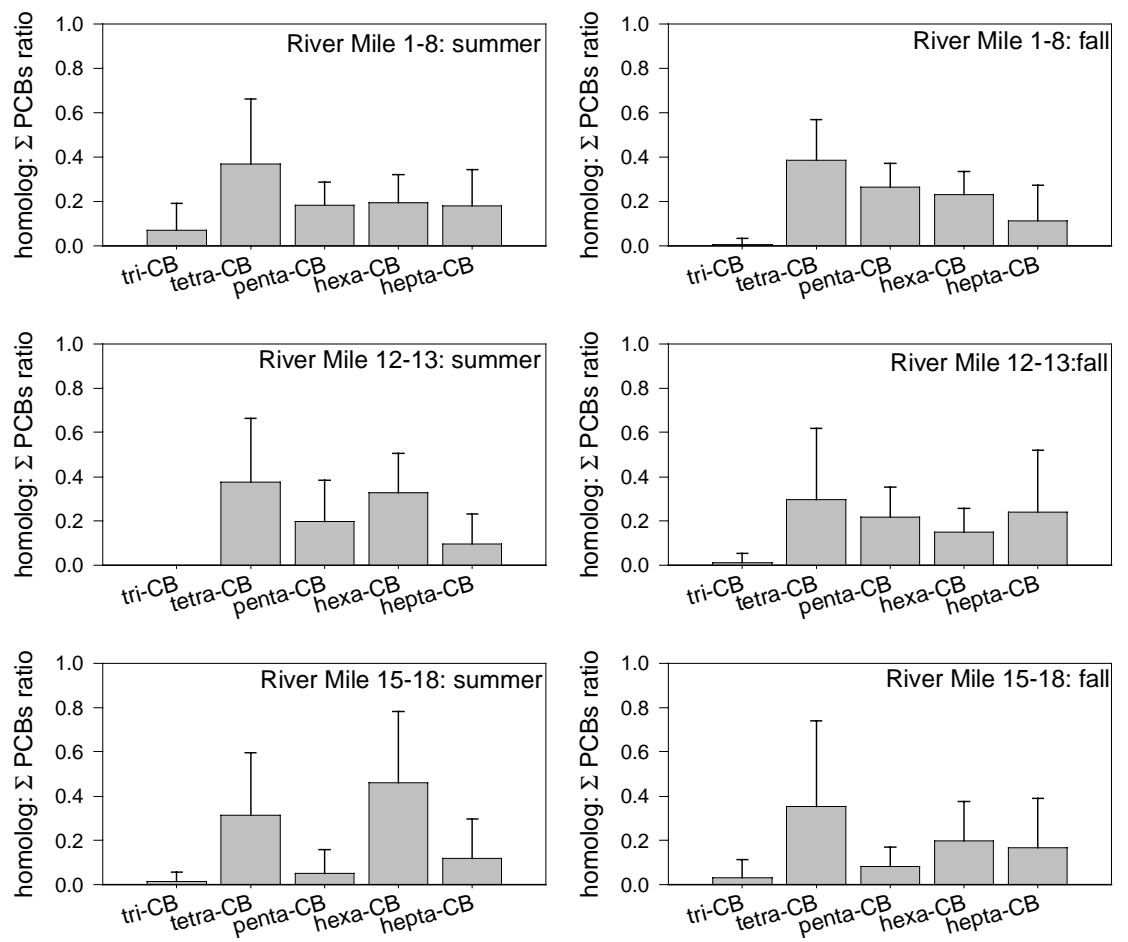
1. Huckins JN, Petty JD, Lebo JA, Orazio CE, Clark RC, Gibson VL. SPMD technology tutorial ( $3^{\text {rd }}$ Edition), vol 2004:The United States Geological Survey, 2002.

2. Meadows JC, Echols KR, Huckins JN, Borsuk FA, Carline RF, Tillitt DE. Estimation of uptake rate constants for PCB congeners accumulated by semipermeable membrane devices and brown trout (Salmo trutta). Environmental Science and Technology 32:1847-1852(1998).

3. Rantalainen A-L, Cretney WJ, Ikonomou MG. Uptake rates of semipermeable membrane devices (SPMDs) for PCDDs, PCDFs, and PCBs in water and sediment. Chemosphere 40:147-158(2000).

4. Petty JD, Huckins JN, Orazio CE, Lebo JA, Clark RC, Gibson VL. Evaluation of the semipermeable membrane devices (SPMD) as a passive in situ concentrator of military organic chemicals in water, Final report, approved for release. Fort Detrick, MD: US Army Medical Research and Material Command, 1997.

5. United States Environmental Protection Agency. National recommended water quality criteria: 2002 EPA-822-R-02-047. Washingtom, DC: Office of Water and Office of Science and Technology, 2002.

6. Fitzpatrick MS. Toxic compounds criteria: 1999-2003 Water quality standards review, issue paper. EQC Meeting, Agenda item B, Rule adoption. Portland, OR: State of Oregon Department of Environmental Quality, 2004. 